7th International Workshop on Astronomy and

Relativistic Astrophysics (IWARA 2016)

International Journal of Modern Physics: Conference Series

Vol. 45 (2017) 1760008 (4 pages)

(C) The Author(s)

DOI: $10.1142 / S 2010194517600084$

\title{
Gamma Ray Bursts and Stellar Evolution
}

\author{
Kauan D. Marquez and Débora P. Menezes \\ Centro de Ciências Físicas e Matemáticas \\ Universidade Federal de Santa Catarina (UFSC) \\ 88.040-900 Florianópolis, Santa Catarina CP 476, Brazil \\ kmarkez@hotmail.com,debora.p.m@ufsc.br
}

Published 15 August 2017

\begin{abstract}
The phenomenon that originates gamma ray bursts (GRBs) remains undefined. In this work the conversion of a hadronic star into a quark star is discussed as one of the possible causes of GRBs. Effective models are used to describe the compact stars and to obtain their equations of state. Macroscopic properties, such baryonic and gravitational masses, of both types of stars are then obtained from the solution of the hydrostatic equilibrium equations. The relation between this values allows to calculate the amount of energy possibly released in this process. The obtained results are then compared to actual GRB observational data, and are within the observational order of magnitude.
\end{abstract}

Keywords: Compact Stars, Gamma Ray Bursts, Effective Models.

PACS numbers: 26.60.+c, 98.70.Rz, 24.10.Jv, 95.30.Tg

\section{Introduction}

Gamma ray bursts (GRBs) are high energy events, known as the brightest electromagnetic events that occur in the universe. They can be distinguished mainly by their duration and released energies in long (LGRBs) and soft (SGRBs) gamma ray bursts. The total energy released in the first few hundred seconds by LGRBs is of the order of $10^{53} \mathrm{erg}$, which is about two orders greater than seen in SGRBs.

Despite many proposed candidates, the phenomenon (or phenomena) that originates GRBs remains undefined. Not long ago ${ }^{1}$ it was proposed that GRBs could be a manifestation of a phase change, from a hadronic to a quark phase inside neutron stars. This idea is derived from the Bodmer-Witten hypothesis, ${ }^{2}$ which states that the ground state of matter is the deconfined quark phase under conditions of extreme densities, giving rise to the possibility of pulsars as strange stars, consisting

This is an Open Access article published by World Scientific Publishing Company. It is distributed under the terms of the Creative Commons Attribution 4.0 (CC-BY) License. Further distribution of this work is permitted, provided the original work is properly cited. 
solely of quarks and leptons. In this work the conversion of a hadronic star into a quark star is discussed as one of the possible causes of GRBs.

\section{Formalism}

A brief summary of the models used in our calculations is given in this section. The nonlinear Walecka model ${ }^{3}$ (NLWM) and the MIT bag model ${ }^{4}$ are used to describe, respectively, the hadronic and the quark phase. A non-interacting lepton gas is included in both descriptions to guarantee the charge neutrality and $\beta$ equilibrium conditions. Also, the stars are assumed to be at $T=0$.

\subsection{Nonlinear Walecka Model}

The lagrangian density of the NLWM is given by

$$
\begin{aligned}
\mathcal{L}= & \sum_{B} \bar{\psi}_{B}\left[\gamma_{\mu}\left(i \partial^{\mu}-M+g_{s B} \phi-g_{v B} V^{\mu}-g_{\rho B} \mathbf{t} \cdot \vec{b}^{\mu}\right)\right] \psi_{B} \\
& -\frac{1}{2} m_{s}^{2} \phi^{2}+\frac{1}{2} m_{v}^{2} V_{\mu} V^{\mu}+\frac{1}{2} m_{\rho}^{2} \vec{b}_{\mu} \cdot \vec{b}^{\mu} \\
& +\frac{1}{2} \partial_{\mu} \phi \partial^{\mu} \phi-\frac{1}{3} \kappa \phi^{3}-\frac{1}{4} \lambda \phi^{4}-\frac{1}{4} \Omega_{\mu \nu} \Omega^{\mu \nu}-\frac{1}{4} \vec{B}_{\mu \nu} \cdot \vec{B}^{\mu \nu}
\end{aligned}
$$

where the sum $\sum_{B}$ extends over the considered baryons, $m_{i}$ and $g_{i B}$ stands for the meson $i=s, v, \rho$ mass and the coupling constant of this meson with the baryon $B$, respectively. The isospin operator is represented by $\mathbf{t}$. The $\phi$ field self-interaction is considered (Boguta-Bodmer terms), $\kappa$ and $\lambda$ stands for their coupling constants.

The calculations are made for hadronic matter constituted (i) only by nucleons and (ii) by the eight lightest baryons. The free parameters of the model are chosen to produce larger masses for the compact star when hyperons are considered, and the three sets selected are NL3, ${ }^{5} \mathrm{NL}^{6}{ }^{6}$ and GM1. ${ }^{7}$

\subsection{MIT Bag Model}

The MIT bag model has been widely used to describe quark matter. This model considers the free quarks inside a bag (represented by the constant $B$ ). The thermodynamic properties are derived from the Fermi gas model, so the EoS are

$$
\begin{aligned}
\varepsilon & =\frac{3}{\pi^{2}} \sum_{q} \int_{0}^{p_{f}} d p p^{2} \sqrt{p^{2}+m_{q}^{2}}+B, \\
P & =\frac{1}{\pi^{2}} \sum_{q} \int_{0}^{p_{f}} d p \frac{p^{4}}{\sqrt{p^{2}+m_{q}^{2}}}-B,
\end{aligned}
$$

where $q=u, d, s, m_{q}$ are the masses $\left(m_{u}=m_{d}=5.5 \mathrm{MeV}, m_{s}=150.0 \mathrm{MeV}\right)$ and $p_{f}=\sqrt[3]{3 \pi^{2} \rho_{q}}$ is the Fermi momentum of the quark $q$. Two bag values are 
chosen, $B^{1 / 4}=148 \mathrm{MeV}$ and $B^{1 / 4}=156 \mathrm{MeV}$. These values are, respectively, at the bottom and in the middle of the stability window presented in Ref. ${ }^{8}$

\section{Results and Discussion}

The EoS given by the effective models are input information to the TolmanOppenheimer-Volkoff equations for relativistic hydrostatic equilibrium (TOV). ${ }^{9}$ Macroscopic properties, such baryonic and gravitational masses, are then obtained from the solution of the TOV and the results are plotted in Fig. 1.
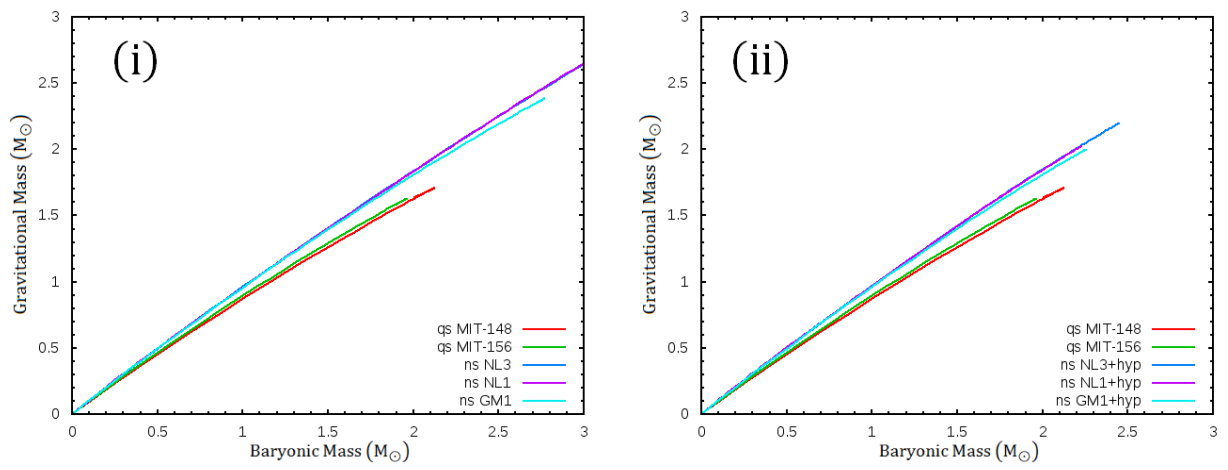

Fig. 1. Baryonic versus gravitational mass for (i) nucleon-only neutron stars and quark stars and (ii) for hyperonic neutron stars and quark stars.

The baryonic mass conservation is taken here as the analogous of the baryonic number conservation, and is assumed during the conversion process. Thus, the released energy in such processes can be easily calculated from the well-known mass-energy relation, where the gravitational masses of the initial (neutron) an final (quark) star are taken for the same baryonic mass. This energy can be given by

$$
\Delta E=\left(M_{n s}-M_{q s}\right) \times 17.88 \times 10^{53} \mathrm{erg},
$$

where $M_{i s}$ stands for the mass of the $i=n, q$ star, in $M_{\odot}$ units. In Fig. 2 we show the released energy during the possible conversion of neutron stars described by all the three parameter sets of the NLWM, with and without the hyperons, to the two quark stars considered.

\section{Conclusion}

To all chosen parameter combinations, the released energy of the conversions are of the order of $10^{53} \mathrm{erg}$, what leads us to conclude that the considered hypothesis of the conversion of a hadronic star into a quark star as one of the possible causes of LGRBs is reasonable. However, a best choice of parameters is still necessary, considering that the results are highly model-sensitive. 

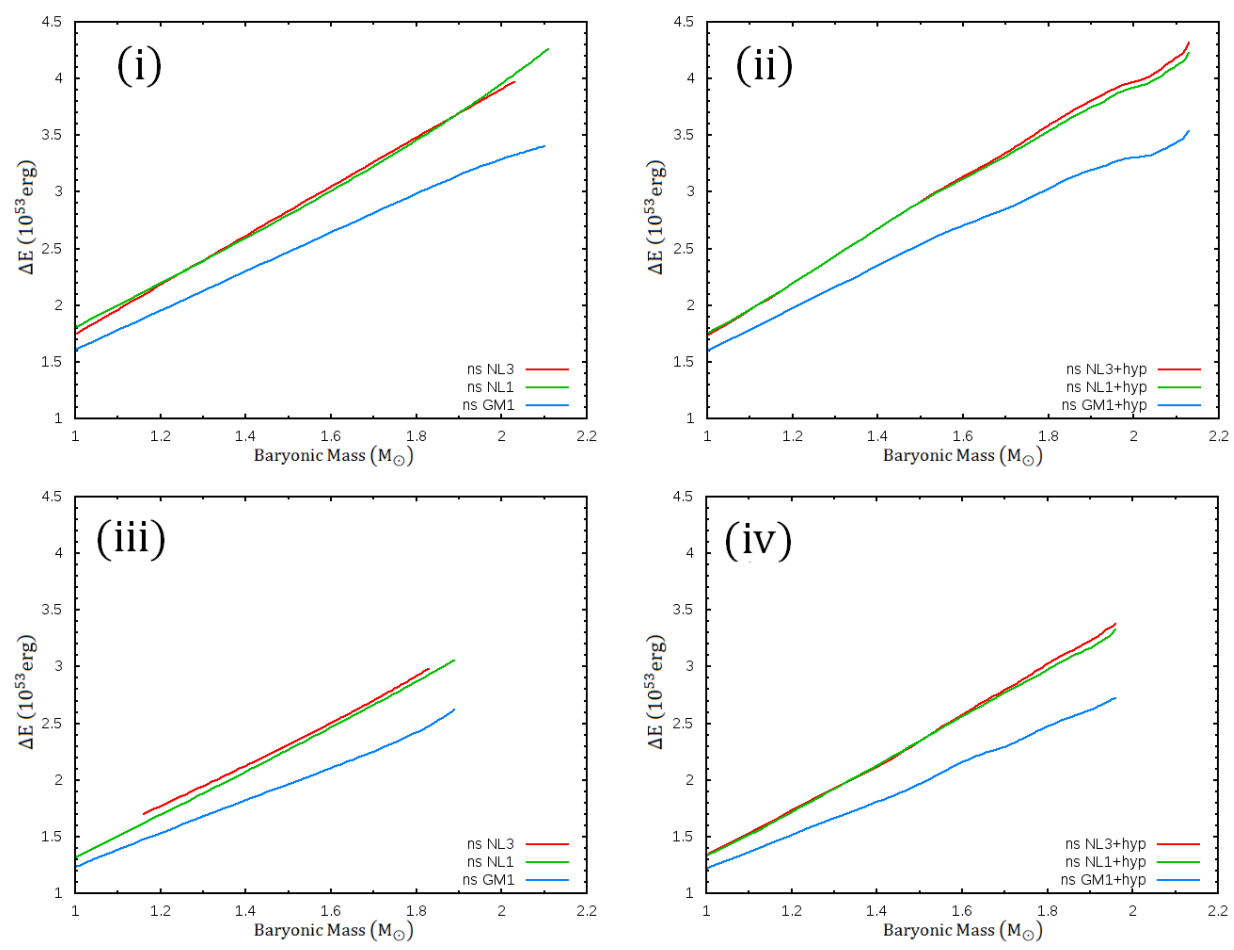

Fig. 2. Energy released in the conversion of (i) nucleon-only neutron stars to quark stars (qs MIT-148), (ii) hyperonic neutron stars to quark stars (qs MIT-148), (iii) nucleon-only neutron stars to quark stars (qs MIT-156) and (iv) hyperonic neutron stars to quark stars (qs MIT-156).

\section{Acknowledgments}

K. D. M. would like to thank the scholarship provided by CAPES and D. P. M. would like to thank the partial support from CNPq.

\section{References}

1. D. P. Menezes, D. B. Melrose, C. Providncia, and K. Wu, Phys. Rev. C 73, 025806 (2006); I. Bombaci and B. Datta, Astrophys. J. 530, L69 (2000).

2. A. R. Bodmer, Phys. Rev. D 4, 1601 (1971); E. Witten, Phys. Rev. D 30, 272 (1984).

3. B. D. Serot and J. D. Walecka, Adv. Nucl. Phys. 16, 1 (1986); J. Boguta and A. R. Bodmer, Nucl. Phys. A 292, 413 (1977).

4. A. Chodos, R. L. Jaffe, K. Johnson, C. B. Thorne, and V. F. Weisskopf, Phys. Rev. D 9, 3471 (1974).

5. G. A. Lalazissis, J. Knig, and P. Ring, Phys. Rev. C 55, 540 (1997).

6. P.-G. Reinhard, Rep. Prog. Phys. 52, 439 (1989).

7. N. K. Glendenning and S. A. Moszkowski, Phys. Rev. Lett. 67, 2414 (1991).

8. J. R. Torres and D. P. Menezes, Europhys. Lett. 101, 42003 (2013).

9. R. C. Tolman, Phys. Rev. 55, 364 (1939); J. R. Oppenheimer and G. M. Volkoff, Phys. Rev. 55, 374 (1939). 